\title{
Structural Studies of FOXC2 Protein and Inhibitor Complexes
}

Shichang Li, Maithri Kashipathy, Maria Castaneda, Jiyong Lee and Hyun-Joo Nam Department of Bioengineering, University of Texas at Dallas

Forkhead C2 (FOXC2) transcription factor, normally regulating vascular endothelial development processes, exhibits abnormally high expression in cancer stem cells, a major culprit for cancer recurrence. It is also associated with endothelial to mesenchymal transition, a crucial process in cancer metastasis. Cancer recurrence, metastasis and angiogenesis are key elements of late stage cancers for which only very limited treatment options are available. Thus, FOXC2 can serve as an ideal target for the new generations of cancer therapeutics. We identified several candidates of FOXC2 inhibitors from helix-mimetics libraries. To understand detailed mechanisms of functional interference by the inhibitors and to optimize the inhibitors through structure-guided design approaches, we initiated structural studies of the human FOXC2 and the inhibitor complexes using X-ray crystallography methods. Crystals of FOXC2 protein in complex with the target DNA and an inhibitor have been obtained, and the crystallization conditions are being optimized. The structure analysis of the FOXC2 will facilitate understanding of its functions and provide basis for the therapeutics development targeting this protein. 